\title{
Immunoglobulin heavy chain expression shapes the $B$ cell receptor repertoire in human $B$ cell development
}

\author{
Eric Meffre, ${ }^{1,2}$ Michèle Milili, ${ }^{2}$ Carla Blanco-Betancourt, ${ }^{2}$ Henedina Antunes, ${ }^{3}$ \\ Michel C. Nussenzweig, ${ }^{1}$ and Claudine Schiff ${ }^{2}$ \\ ${ }^{1}$ Laboratory of Molecular Immunology, The Rockefeller University, \\ Howard Hughes Medical Institute, New York, New York, USA \\ ${ }^{2}$ Centre d'Immunologie de Marseille-Luminy, CNRS-INSERM-Université de la Méditerranée, \\ Campus de Luminy, Marseille, France \\ ${ }^{3}$ Fundacao Faculdade Federal de Ciencias, Medicas de Porto Alegre, Portugal
}

Address correspondence to: Eric Meffre, The Rockefeller University, 1230 York Avenue, New York, New York 10021, USA. Phone: (212) 327-8098; Fax: (212) 327-8370; E-mail: meffree@mail.rockefeller.edu.

Received for publication April 16, 2001, and accepted in revised form August 13, 2001.

\begin{abstract}
Developing B cells must pass a series of checkpoints that are regulated by membrane-bound Ig $\mu$ through the $\operatorname{Ig} \alpha-\operatorname{Ig} \beta$ signal transducers. To determine how Ig $\mu$ expression affects B cell development and $\mathrm{Ab}$ selection in humans we analyzed Ig gene rearrangements in pro-B cells from two patients who are unable to produce Ig $\mu$ proteins. We find that Ig $\mu$ expression does not affect $V_{H}, D$, or $J_{H}$ segment usage and is not required for human $I g \kappa$ and $I g \lambda$ recombination or expression. However, the heavy and light chains found in pro-B cells differed from those in peripheral B cells in that they showed unusually long CDR3s. In addition, the IgK repertoire in Ig $\mu$-deficient pro-B cells was skewed to downstream $J \kappa s$ and upstream $V \kappa s$, consistent with persistent secondary $V(D) J$ rearrangements. Thus, Ig $\mu$ expression is not required for secondary $\mathrm{V}(\mathrm{D}) \mathrm{J}$ recombination in pro-B cells. However, B cell receptor expression shapes the $\mathrm{Ab}$ repertoire in humans and is essential for selection against $\mathrm{Ab}$ 's with long CDR3s.

J. Clin. Invest. 108:879-886 (2001). DOI:10.1172/JCI200113051.
\end{abstract}

\section{Introduction}

Expression of immunoglobulin heavy chains $(\operatorname{Ig} \mu)$ in pro-B cells induces pre- $\mathrm{B}$ cell development by assembly of a pre-B cell receptor (pre-BCR), which is a complex of Ig $\mu$, surrogate light chains $(\psi \mathrm{L})$, and two signal-transducing proteins, $\operatorname{Ig} \alpha$ and $\operatorname{Ig} \beta(1-6)$. Pre-BCR expression induces proliferative expansion and downregulation of recombinase-activating genes (RAG1 and RAG2), thereby ensuring allelic exclusion while selecting clones of cells with productive $\mathrm{VDJ}_{\mathrm{H}}$ rearrangements (7). Mutations in $m I g \mu$, $\psi L$, or $I g \alpha / \operatorname{Ig} \beta$ genes that disrupt pre-BCR assembly impede $B$ cell development at the pro-B cell stage in mice and humans (8-15).

Pre-BCR assembly is dependent on interaction between $V_{\mathrm{H}}$ variable regions and $\psi \mathrm{L}$, and in the mouse the $\mathrm{IgH}$ repertoire is selected in part on the basis of this interaction (16-18). It has been proposed that $\mathrm{V}_{\mathrm{H}}$ domains that pair well with $\psi \mathrm{L}$ are positively selected at the pre- $\mathrm{B}$ cell stage, whereas $\mathrm{V}_{\mathrm{H}}$ domains that pair poorly with $\psi \mathrm{L}$ are not. However, selection differs in mouse fetal and adult $B$ cell development such that $V_{H} S$ that are counterselected in the adult are prominent in the fetal repertoire (18).

In contrast to mice, there is no apparent difference in the $\mathrm{V}_{\mathrm{H}}$ repertoire between pro- $\mathrm{B}$, pre- $\mathrm{B}$, or fetal $\mathrm{B}$ cells and adult mature $B$ cells in the human (19-30). However, there is selection for $\mathrm{IgH}$ genes during human $\mathrm{B}$ cell development as determined by the length of the third complementary determining region (CDR3). Proand pre- $\mathrm{B}$ cells from adult bone marrow have longer CDR3s than mature B cells (31-33).

Following successful IgH assembly and pre-BCR expression, $\mathrm{V}(\mathrm{D}) \mathrm{J}$ recombination is targeted to the light chain (IgL) genes (34-38). Those cells that produce inframe IgL chains test their newly synthesized Igs for selfreactivity. In the mouse, B cells that produce self-reactive receptors are either deleted or arrested in development and undergo receptor editing (39-45). In contrast, less is known about receptor selection and the role of the BCR in regulating B cell development in humans. Here we report on the role of the BCR in Ig repertoire selection in two patients with different mutations in the I $g \mu$ gene that impairs BCR assembly.

\section{Methods}

Patient samples and cell preparation. Bone marrow samples were obtained from two I $g \mu$-deficient patients with either a homozygous cytidine insertion in the $I g \mu$ gene $\left(I g \mu^{-/-}\right)$or with a homozygous deletion of the Ig $\mu$ locus $\left(\operatorname{Ig} \mu^{\Delta}\right)$ (see Results) $(13,46,47)$ (C. Schiff, unpublished observations). Samples were obtained when the $\operatorname{Ig} \mu^{\Delta}$ patient was 2 years old, the $I g \mu^{-/-}$patient was 4 years old, and her $I g \mu^{+/-}$brother (control) was 9 months old. The parents gave informed consent for this study. Bone marrow mononuclear cells were isolated by Ficoll gradients and $\mathrm{CD} 34^{+} \mathrm{CD} 19^{+}$pro-B cells were sorted on a 
FACSVantage after labeling with FITC anti-CD34 and phycoerythrin anti-CD19 mAb's (Beckman Coulter, Brea, California, USA).

$R N A$ and RT-PCR. Total RNA was extracted from $10^{4}-10^{5}$ purified cells using TRIzol Reagent (Life Technologies Inc., Rockville, Maryland, USA). RNA was reverse transcribed with Superscript II (Life Technologies Inc.) according to the manufacturer's instructions. For RT-PCR reactions, cDNA was amplified for 25 (actin), $35\left(\mathrm{~V}_{\mathrm{H}^{-}} \mathrm{C}_{\mu}\right)$, or $38\left(\mathrm{~V}_{\mathrm{K}^{-}} \mathrm{C}_{\mathrm{K}}\right)$ cycles of 30 seconds at $94^{\circ} \mathrm{C}, 30$ seconds at $60^{\circ} \mathrm{C}$, and 30 seconds at $72^{\circ} \mathrm{C}$, or for 40 cycles $\left(\mathrm{V}_{\lambda}-\mathrm{C}_{\lambda}\right)$ of 30 seconds at $94^{\circ} \mathrm{C}, 30$ seconds at $55^{\circ} \mathrm{C}$, and 30 seconds at $72^{\circ} \mathrm{C}$, with a final 10 -minute extension at $72^{\circ} \mathrm{C}$ using Hot Star Taq DNA polymerase (QIAGEN Inc., Valencia, California, USA) and the following primers: $V_{\lambda}$ consensus sense, $5^{\prime} \mathrm{GGG}(\mathrm{G} / \mathrm{A}) \mathrm{TC}(\mathrm{T} / \mathrm{C}) \mathrm{CT}$ GA(C/T/G)CG(A/C/G)TTCTCTGG(C/G)TCC3'; $\mathrm{C}_{\lambda}$ antisense, 5'CACAC(T/C)AGTGTGGCCTTGTTGGCTTG3'. $\mathrm{V}_{\mathrm{H}} 1, \mathrm{~V}_{\mathrm{H}} 3, \mathrm{~V}_{\mathrm{H}} 4, \mathrm{C}_{\mu}, \mathrm{V}_{\mathrm{K}}$ consensus and $\mathrm{C}_{\mathrm{K}}$ primers were described previously $(48,49)$. RT-PCR products were analyzed on $2 \%$ agarose gels and visualized by adding 0.3 pmol of ${ }^{32}$ PdATP to the PCR reaction.

Cloning and sequencing. PCR products were gel-purified (Qiaquick; QIAGEN Inc.) and cloned into TA vectors (Invitrogen, Carlsbad, California, USA). Doublestranded DNA sequences were obtained using antisense $C_{\mu}, C_{\kappa}$, or $C_{\lambda}$ primers and Dye Terminator Cycle Sequencing (Applied Biosystems, Foster City, California, USA). Sequences were analyzed by comparison with Ig basic alignment search tool (BLAST). IgH CDR3 length was determined by counting amino acid residues between positions 94 and 102 (conserved tryptophan in all $\mathrm{J}_{\mathrm{H}}$ segments) and $\mathrm{D}$ segments were identified following the criteria of Corbett et al. (50). IgK and Ig $\lambda$ CDR3 length included amino acids between conserved cystein 88 and the phenylalanine residue embedded in $J_{\kappa}$ or $J_{\lambda}(51)$. Nontemplate $(\mathrm{N})$ nucleotides (52) found at $V_{\kappa}-J_{\kappa}$ or $V_{\lambda}-J_{\lambda}$ junctions were counted while template-dependent palindromic $(\mathrm{P})$ nucleotides (53) were excluded. Differences in gene distribution were analyzed with $\chi^{2}$ tests (Cochran-Mantel-Haenszel test) adjusted by the Bonferroni method for multiple testing, and they were considered significant when $P$ values were less than or equal to 0.05 .

\section{Results}

IgH and IgL transcription is independent of Ig $\mu$ expression. Two patients with agammaglobulinemia and $\operatorname{IgH}$ mutations were studied. Ig $\mu^{-/-}$has a cytidine insertion in the CH1 exon of the Ig $\mu$ gene that leads to a frameshift and the inability to produce Ig $\mu$ products $(13,46,47)$. Ig $\mu^{\Delta}$ has a deletion in the Ig locus from $3^{\prime}$ of the diversity (D) region to $\operatorname{Ig} \gamma 2$, with all junction (J) segments and $\operatorname{Ig} \mu, \operatorname{Ig} \delta, \operatorname{Ig} \gamma \beta$, and $\operatorname{Ig} \gamma 1$ genes missing (C. Schiff, unpublished observations).

Flow cytometric analysis of bone marrow from $I g \mu^{-/-}$ and $I g \mu^{\Delta}$ patients revealed that in both cases B cell differentiation was arrested at the $\mathrm{CD} 34^{+} \mathrm{CD} 19^{+}$pro- $\mathrm{B}$ cell stage (Figure 1a) $(12,13,46,47)$. To characterize Ig expression in Ig $\mu$-deficient pro-B cells, transcripts for heavy and light chain genes were amplified by semiquantitative RT-PCR from sorted $\mathrm{CD} 34^{+} \mathrm{CD} 19^{+}$ pro-B cells from $I g \mu^{-/-}, \operatorname{Ig} \mu^{\Delta}$, and a control sibling (Figure 1). As expected, $\mathrm{V}_{\mathrm{H}^{-}} \mathrm{C}_{\mu}$ mRNA was missing in $I g \mu^{\Delta}$ pro-B cells where the entire I $g \mu$ locus was deleted (Figure 1b). In contrast, $I g \mu^{-/-}$and control pro-B cells showed similar levels of $\mathrm{V}_{\mathrm{H}^{-}} \mathrm{C}_{\mu}$ transcripts revealing that the absence of Ig $\mu$ protein does not affect the $\operatorname{Ig} \mu$ gene expression in humans (Figure 1b).

\footnotetext{
Figure 1

Immunoglobulin rearrangements in human pro-B cells. (a) B cell precursors in sibling control (left), $\lg \mu^{-/-}$(middle), and $\lg \mu^{\Delta}$ (right) bone marrow. $\mathrm{CD} 34^{+} \mathrm{CD} 19^{+}$pro-B cells from control and both $\lg \mu$-deficient patients were sorted as gated. (b) Heavy and light chain Ig gene expression in human pro-B cells. RNA from FACS-sorted CD $34^{+} \mathrm{CD} 19^{+}$pro-B cells from both $I g \mu$-deficient patients and control was analyzed by semiquantitative RT-PCR using $5^{\prime}$ consensus $V_{H}, V_{\kappa}$, or $V_{\lambda}$ and $3^{\prime} C_{\mu}, C_{\kappa}$, or $C_{\lambda}$ primers, respectively, and visualized by ${ }^{32}$ PdATP incorporation. "Neg." denotes a negative control without cDNA for RT-PCR reactions. Actin RT-PCR was used as mRNA loading control. Serial fivefold dilutions of cDNA are shown.
}

a
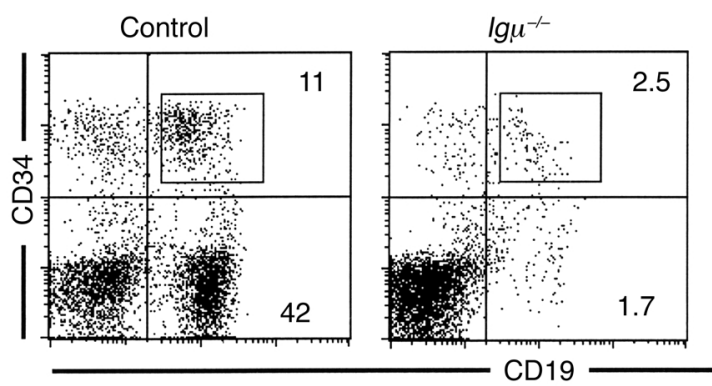

$\lg \mu^{-1-}$

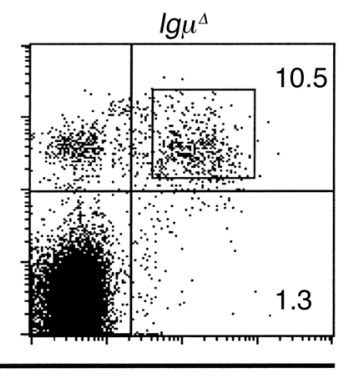

b

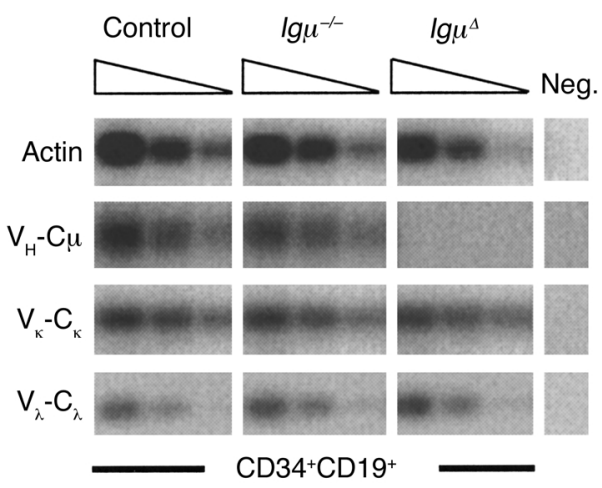




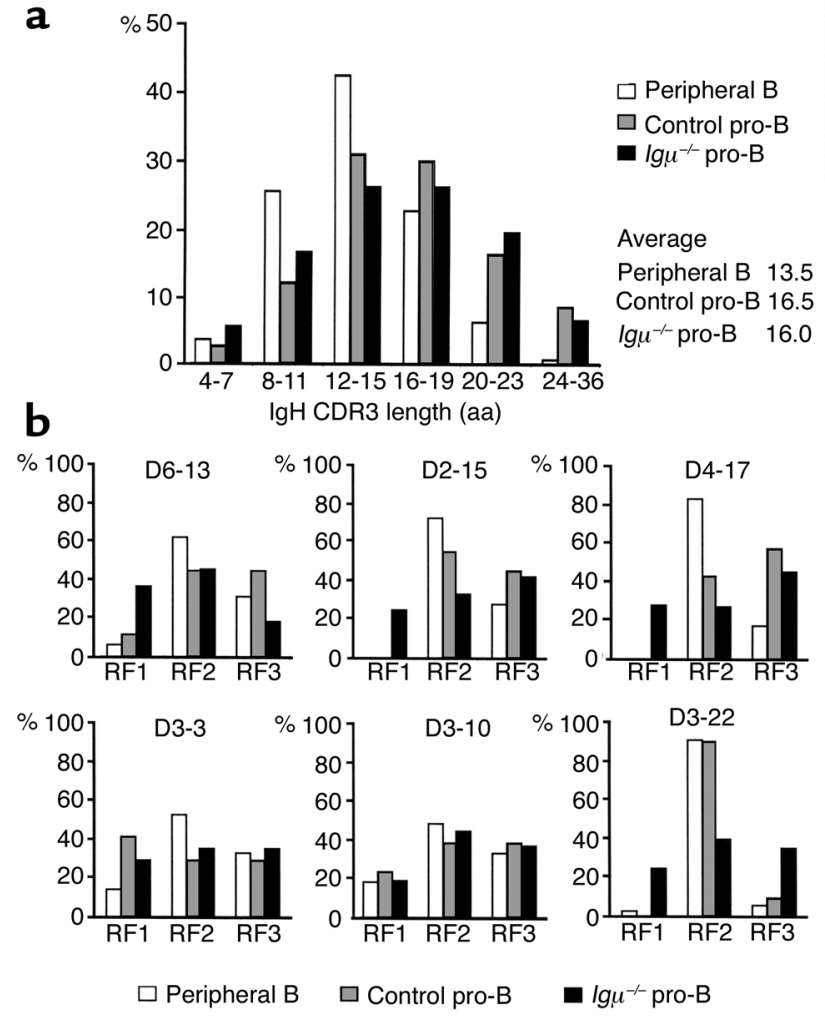

Light chain gene transcripts were found at similar levels in sorted control or Ig $\mu$-deficient pro-B cells (Figure 1b), but they were not amplified from $I g \mu^{-/-}$ total bone marrow cells that contain few pro-B cells $(46,47)$. We conclude that light chain genes can be recombined and expressed in the absence of $\operatorname{Ig} \mu$ in human pro-B cells.

Ig $\mu$-independent $V_{H} D$, and $J_{H}$ gene segment usage. To determine whether Ig $\mu$ expression is required for $V_{H}, D$, or $J_{H}$ segment selection, IgH genes from the three major $V_{H}$ families, $V_{H} 1, V_{H} 3$, and $V_{H} 4$, were cloned and sequenced. $V_{H}, D$, and $J_{H}$ repertoire analysis revealed no statistically significant differences between $I g \mu^{-/}$pro-B cells, control pro-B cells, and peripheral B cells $(19,20,26-30)$ (see supplemental data 1, www.jci.org/cgi/content/full/$108 / 06 / 879 / D C 1)$. Of $27 \mathrm{D}$ genes in humans, only the D2-2 gene segment was over-represented in $I g \mu^{-/-}$and control pro-B cells $(P=0.02)(31,50)$ (see supplemental data 2, www.jci.org/cgi/content/full/108/06/879/DC2).

\section{Figure 2}

IgH CDR3 characteristics in pro-B cells. (a) $V_{H} D J_{H} C D R 3$ length in 350 peripheral $B$ cell (white bars), 117 in-frame control pro-B (gray bars), and $197 / g \mu^{-/-}$(black bars) individual sequences. CDR3 length in amino acids (aa) is indicated below. The average CDR3 length for peripheral $\mathrm{B}$, control pro- $\mathrm{B}$, and $I g \mu^{-/-}$pro-B cells was $13.5,16.5$, and 16.0 amino acids, respectively. (b) $D$ reading frame usage in $\lg \mathrm{H}$ $\mathrm{CDR3s}$ from peripheral $\mathrm{B}$, control, and $/ g \mu^{-/-}$pro-B cells. The three RF uses reported by Corbett et al. (50) for some commonly used $D$ gene segments are represented. D3-3 and D3-10 encode no intragenic stop codons whereas D6-13, D2-15, D4-17, and D3-22 sequences using RF1 display stop codons.

Thus, the pre-BCR is not essential for $\mathrm{V}_{\mathrm{H}}$ selection, and intrinsic genetic factors are responsible for specific $V_{H}$, $\mathrm{D}$, and $\mathrm{J}_{\mathrm{H}}$ gene usage in human $\mathrm{B}$ cells.

IgH CDR 3 selection by I $g \mu$. To determine whether Ig $\mu$ expression influences CDR3 selection, CDR3 length and amino acid composition were analyzed in $I g \mu^{-/-}$ pro-B cells and compared with control pro-B and peripheral B cells. About two-thirds of IgH genes were out of frame in $I g \mu^{-/-}$pro-B cells, confirming the absence of Ig $\mu$-mediated selection in these cells (Table 1) (20). In contrast, two-thirds of $\operatorname{IgH}$ CDR3s were found to be in-frame in control $\mathrm{CD} 34^{+} \mathrm{CD} 19^{+}$precursor cells, suggesting early Ig $\mu$-mediated positive selection of a subpopulation of $\mathrm{CD} 34^{+} \mathrm{CD} 19^{+}$cells that express Ig $\mu$ (Table 1). We compared $\operatorname{IgH}$ genes expressed by $I g \mu^{-/}$pro-B cells to the in-frame IgH genes expressed by control CD $34^{+} \mathrm{CD} 19^{+}$cells and found an average CDR3 length of 16.0 and 16.5 amino acids, respectively, whereas peripheral $\mathrm{B}$ cells showed an average CDR3 length of 13.5 amino acids (Figure 2a). D-D fusions that increase the length of CDR3 were found in about $2 \%$ (4 of 198) of the IgH sequences from $\operatorname{Ig}^{-/-}$ pro-B cells and in $2.6 \%$ (3 of 117) of those from control $\mathrm{CD} 34^{+} \mathrm{CD} 19^{+}$cells, but were absent in peripheral B cells $(49,50)$ (data not shown). We conclude that IgH CDR3 length is not selected in early B cell precursors and that long CDR3s and D-D fusions are counterselected during late stages of $\mathrm{B}$ cell development.

D segments can be used in three different reading frames, but in humans, RF1 tends to encode stop codons, RF2 usually encodes glycine residues, and hydrophilic amino acids and RF3 is biased to encode hydrophobic sequences (50). D segments in RF1 are

Table 1

CDR3 frame analysis in CD $34^{+}$CD $19^{+}$pro-B cells

\begin{tabular}{|c|c|c|c|c|c|c|c|c|}
\hline \multirow{3}{*}{ CDR3 } & \multicolumn{3}{|c|}{ Control } & \multicolumn{3}{|c|}{$\lg \mu^{-/-}$} & \multirow{2}{*}{\multicolumn{2}{|c|}{$\begin{array}{l}\lg \mu^{\Delta} \\
\text { Frame }\end{array}$}} \\
\hline & \multicolumn{2}{|c|}{ Frame } & \multicolumn{4}{|c|}{ Frame } & & \\
\hline & Out & $\ln$ & Stop codon ${ }^{A}$ & Out & In & Stop codon ${ }^{A}$ & Out & In \\
\hline $\mathrm{H}$ & 69 (33\%) & 141 (67\%) & $11(7.8 \%)$ & 143 (73\%) & $54(27 \%)$ & 24 (44\%) & - & - \\
\hline$\kappa$ & $10(45 \%)$ & $12(55 \%)$ & & $21(55 \%)$ & 17 (45\%) & & $25(68 \%)$ & $12(32 \%)$ \\
\hline$\lambda$ & 11 (55\%) & $9(45 \%)$ & & $6(60 \%)$ & $4(40 \%)$ & & 15 (54\%) & $13(46 \%)$ \\
\hline
\end{tabular}

AStop codon frequency is calculated among in-frame CDR3s. 
a
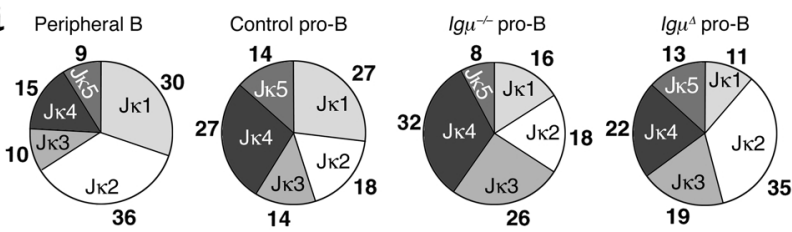

b
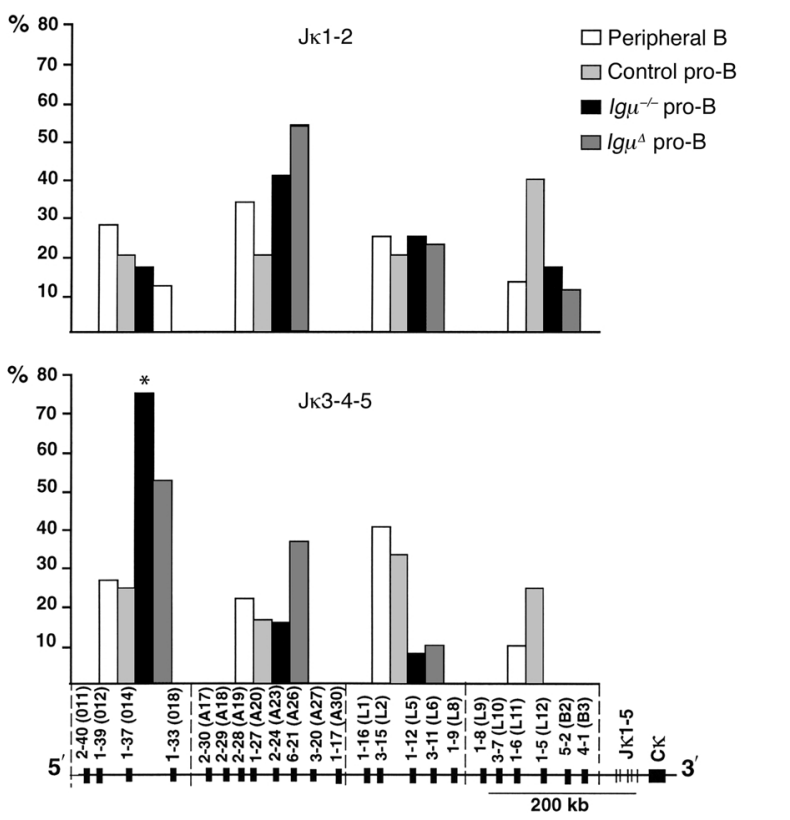

normally under-represented in peripheral B cells and were counterselected in control CD $34^{+} \mathrm{CD} 19^{+}$cells (Figure $2 \mathrm{~b}$, top and bottom row, and Table 1). In contrast, there was neither RF selection nor stop codon counterselection in $I g \mu^{-/-}$pro-B cells (Figure $2 \mathrm{~b}$, top and bottom row, and Table 1). D3-3 or D3-10 genes that do not contain intragenic stop codons in RF1 were used in all three RFs in $I g \mu^{-/-}$, control CD $34^{+} \mathrm{CD} 19^{+}$, or peripheral $\mathrm{B}$ cells (Figure $2 \mathrm{~b}$, middle row). In addition, hydrophilic (RF2) and hydrophobic (RF3) Ds were used equally in Ig $\mu^{-/-}$and control CD $34^{+} \mathrm{CD} 19^{+}$cells whereas RF2 was favored in peripheral B cells (Figure $2 \mathrm{~b})(31,50)$. However, D3-22 RF usage was already selected in control $\mathrm{CD} 34^{+} \mathrm{CD} 19^{+}$cells since RF3 was clearly counterselected (and/or RF2 positively selected) when functional Ig $\mu$ chains were generated (Figure 2b, bottom row). Thus, IgH CDR3s containing stop codons are counterselected in early B cell precursors whereas CDR3s with hydrophilic or hydrophobic RF are not.

\section{Figure 4}

Igא CDR3 characteristics in pro-B cells. (a) Igא CDR3 length in amino acids and (b) $\mathrm{N}$ nucleotide (nt) addition in peripheral $\mathrm{B}$ cells (white bars), control pro-B (light gray bars), Ig $\mu^{-/-}$(black bars), and $\lg \mu^{\Delta}$ (dark gray bars) pro-B cells. For determination of $\mathrm{N}$ nucleotide addition, $P$ nucleotides were not included. The average CDR3 length and $\mathrm{N}$ nucleotide addition for peripheral B, control pro-B, $\lg \mu^{-/-}$, and $\lg \mu^{\Delta}$ pro-B cells was 9.0, 10.2, 11.2, and 11.1 amino acids and 1.0, 3.6, 7.3 , and 6.8 nucleotides, respectively.

\section{Figure 3}

Ig $\kappa$ light chain repertoire in pro- $B$ cells. (a) $J_{\kappa}$ usage in 108 peripheral B, 22 control pro-B, $38 \lg \mu^{-/-}$, and $37 \lg \mu^{\Delta}$ pro-B $V_{\mathrm{K}} J_{\mathrm{K}}$ individual sequences. Percentages of $J_{\kappa}$ usage are indicated. (b) $V_{\kappa}$ usage in upstream $J \kappa 1$ and $J \kappa 2$ (JK1-2; top) and downstream $J \kappa 3$, J $\kappa 4$, and $J \kappa 5$ (JK3-4-5; bottom) rearrangements of peripheral B cells (white bars), control pro-B (light gray bars), Ig $\mu^{-/-}$(black bars), and $\lg \mu^{\Delta}$ (darkgray bars) pro-B cells. The $V_{\kappa}$ genes are subdivided in four groups on the locus (84). The percentages of each $V_{\kappa}$ group are indicated on the $y$ axis. ${ }^{*}$ Statistically significant difference $(P<0.001)$.

Ongoing IgK recombination in Ig $\mu$-deficient pro-B cells. To characterize light chain gene expression in human pro-B cells, we amplified and sequenced Igא mRNAs from $I g \mu^{-/-}$and $I g \mu^{\Delta}$ pro-B cells and compared them with those of control pro-B and peripheral B cells (49). We found that IgK mRNAs expressed in $I g \mu^{-/-}$and $I g \mu^{\Delta}$ pro-B cells showed decreased $J \kappa 1$ and increased downstream $J \kappa 3$ usage when compared with normal B cell controls (Figure $3 a)$. In addition, $J \kappa 3-4-5$ segments from $\operatorname{Ig} \mu^{-/-}$and $I g \mu^{\Delta}$ pro-B cells were preferentially combined with upstream $V \kappa s(P=0.005$, Figure $3 \mathrm{~b})$ whereas there was no such bias in the $J \kappa 3-4-5$ IgK mRNAs from normal pro-B cell and peripheral B cell controls. In contrast, there was no bias in $V \kappa$ gene usage for Igא genes using JK1-2 segments in patients and controls (Figure $3 \mathrm{~b}$ ). We conclude that in the absence of Ig $\mu$ there is a shift in the IgK repertoire to downstream $J_{k} s$ and upstream $V_{\kappa} s$ consistent with secondary IgK rearrangement in pro-B cells.

IgK CDR3 analysis revealed that the ratio of productive to nonproductive $V_{\kappa}-J_{\kappa}$ joints in human pro-B cells was similar to that reported for $m I g \mu$-deficient mouse $(\mu \mathrm{MT})$ pro-B cells (54). IgK CDR3 length was increased in all pro-B cell samples when compared with peripheral B cells (Table 1 and Figure 4a). We found that Igא CDR3s from control pro-B cells and from the patients had an average of $10.2,11.2$, or 11.1 amino acids where-

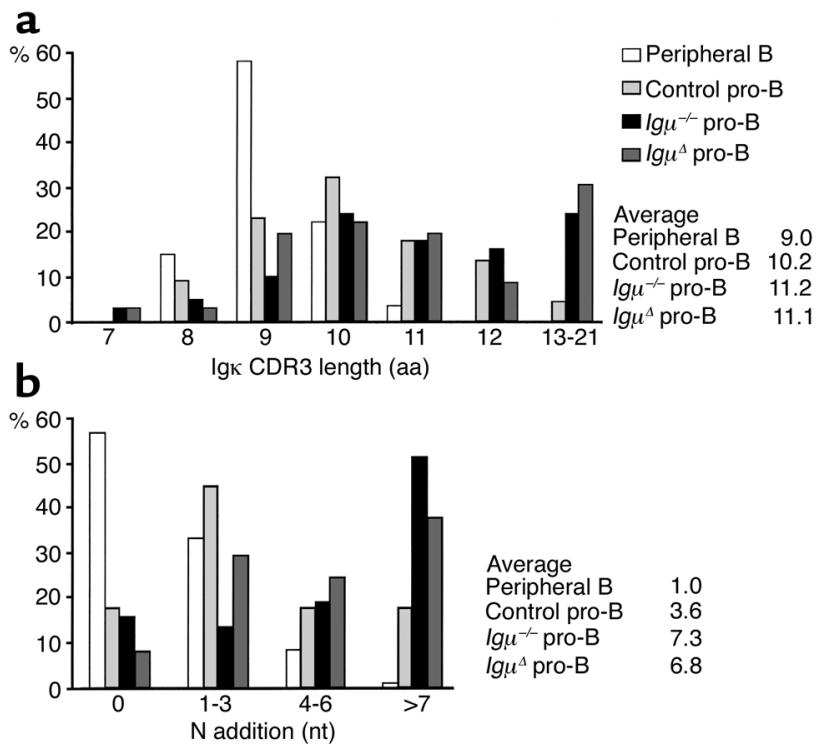



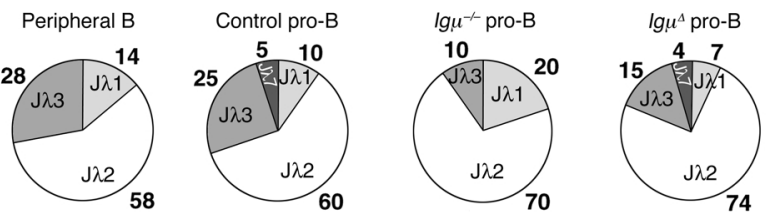

b

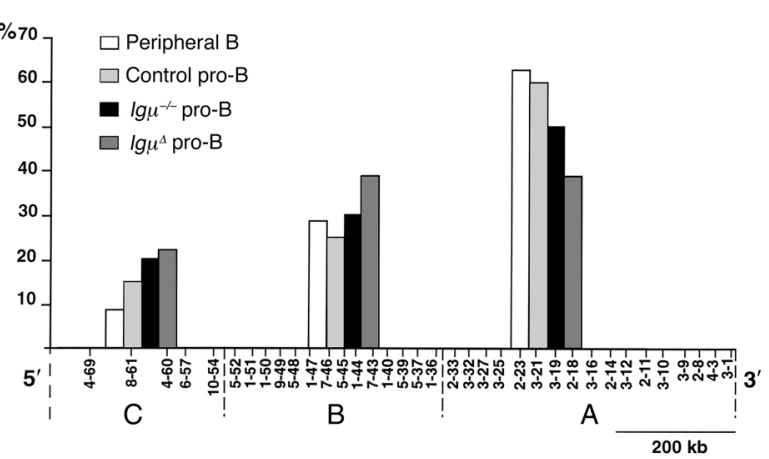

as IgK CDR3 from peripheral B cells were only 9.0 amino acids long (Figure 4a). The increased CDR3 length in pro-B cells was due to terminal deoxynucleotidyl transferase-catalyzed (TdT-catalyzed) addition of template-independent $(\mathrm{N})$ nucleotides and not to template-dependent $\mathrm{P}$ nucleotides (Figure $4 \mathrm{~b}$ ). On average, 3.6, 7.3, and 6.8 nucleotides were added by TdT to $\operatorname{Ig} \kappa$ gene CDR3s in control, $\operatorname{Ig} \mu^{-/-}$, and $\operatorname{Ig} \mu^{\Delta}$ pro-B cells whereas only $1 \mathrm{~N}$ nucleotide was found in $\operatorname{Ig} \kappa$ genes expressed by peripheral B cells (Figure $4 b$ ). We conclude that pro-B cells produce IgK genes with unusually long CDR3s.

Ig $\lambda$ repertoire of Ig $\mu$-deficient pro- $B$ cells. To determine whether $I g \lambda$ genes expressed in $I g \mu$-deficient pro-B cells displayed features similar to $\operatorname{Ig} \kappa$ genes we analyzed the $\operatorname{Ig} \lambda$ repertoire. Although the human $\operatorname{Ig} \lambda$ locus should allow deletional replacement of $V J_{\lambda s}$ by secondary recombination (55), we found no significant increases in either downstream $J_{\lambda}$ or distal $V_{\lambda}$ segment usage in $I g \mu^{-/-}$and $I g \mu^{\Delta}$ pro-B cells. In fact, there was a decrease in downstream $J_{\lambda} 3$ segment usage (56, 57) (Figure 5, $a$ and $b$ ).

$\operatorname{Ig} \lambda$ genes expressed in control, $\operatorname{Ig} \mu^{-/-}$, and $\operatorname{Ig} \mu^{\Delta}$ pro$B$ cells resembled $I g \kappa$ genes in that they showed a similar ratio of in-frame and out-of-frame sequences and long CDR3s, resulting from addition of $\mathrm{N}$ nucleotides by TdT (Figure 6, a and b, and Table 1). The average number of $\mathrm{N}$ nucleotides in $\operatorname{Ig} \lambda \mathrm{CDR} 3 \mathrm{~s}$ from control, $I g \mu^{-/-}$, and $I g \mu^{\Delta}$ pro-B cells was 3.9, 5.0, and 6.5 as compared with 1.2 for mature B cells (Fig-

\section{Figure 6}

$\operatorname{Ig} \lambda$ CDR3 characteristics in pro-B cells. (a) $\lg \lambda$ CDR3 length in amino acids and (b) $\mathrm{N}$ nucleotide addition in peripheral $\mathrm{B}$ cells (white bars), control pro-B (light gray bars), Ig $\mu^{-/-}$(black bars), and $/ g \mu^{\Delta}$ (dark gray bars) pro- $B$ cells. The average $C D R 3$ length and $N$ nucleotide addition for peripheral B, control pro-B, $\lg \mu^{-1-}$, and $\lg \mu^{\Delta}$ pro-B cells was $10.5,11.2,11.4$, and 11.6 amino acids and $1.2,3.9,5.0$, and 6.5 nucleotides, respectively.

\section{Figure 5}

Ig $\lambda$ light chain repertoire in pro- $B$ cells. (a) $\mathrm{J} \lambda$ usage in 163 peripheral B, 20 control pro-B, $10 / g \mu^{-/-}$, and $28 / g \mu^{\Delta}$ pro-B $V_{\lambda} J_{\lambda}$ individual sequences. Percentages of $J_{\lambda}$ usage are indicated. (b) $V_{\lambda}$ usage in peripheral B cells (white bars), control pro-B (light gray bars), Ig $\mu^{-/-}$ (black bars), and $l g \mu^{\Delta}$ (dark gray bars) pro-B cells. The $V_{\lambda}$ locus is shown clustered into three groups $-A, B$, and $C-$ of $V_{\lambda}$ genes (84). The percentages of each $V_{\lambda}$ group are indicated on the $y$ axis.

ure $6 \mathrm{~b})$. We conclude that the $I g \lambda$ genes expressed by $\operatorname{Ig} \mu^{-/-}$and $\operatorname{Ig} \mu^{\Delta}$ pro-B cells differ from $\operatorname{Ig} \kappa$ genes in that they show no signs of secondary recombination, but they resemble Ig $\kappa$ genes in that they display long CDR3s with extensive $\mathrm{N}$ addition.

\section{Discussion}

The absence of Ig $\mu$ in $I g \mu^{-/}$and $\operatorname{Ig} \mu^{\Delta}$ patients provided an opportunity to study the role of Ig $\mu$ in selecting the $\mathrm{Ab}$ repertoire in humans. We found no significant differences in the Ig heavy chain gene $V_{H}, D$, or $J_{H}$ repertoire between Ig-deficient pro-B cells, control pro-B cells, and normal peripheral $\mathrm{B}$ cells. These findings are in agreement with cell-sorting experiments in which normal pro$B$, pre- $B$, and immature $B$ cell repertoires were compared with that of peripheral B cells (19-21,31). Thus, $V_{H}, D$, and $J_{H}$ segment usage in humans is independent of Ig $\mu$ expression and is likely to be a function of intrinsic genetic elements controlling $V_{H}, D$, or $J_{H}$ gene accessibility and recombination. In contrast, IgH CDR3 length appears to be selected throughout $\mathrm{B}$ cell development starting with $\mathrm{CD} 34^{-} \mathrm{CD} 19^{+} \mathrm{IgM}^{-}$pre-B cells (33). Our analysis of $I g \mu^{--}$and control $\mathrm{CD} 34^{+} \mathrm{CD} 19^{+}$cells showed that Ig $\mu$ expression is not involved in IgH CDR3 length and hydrophilic RF selection in the human at the pro-B cell stage. However, in-frame $\operatorname{IgH}$ genes without stop
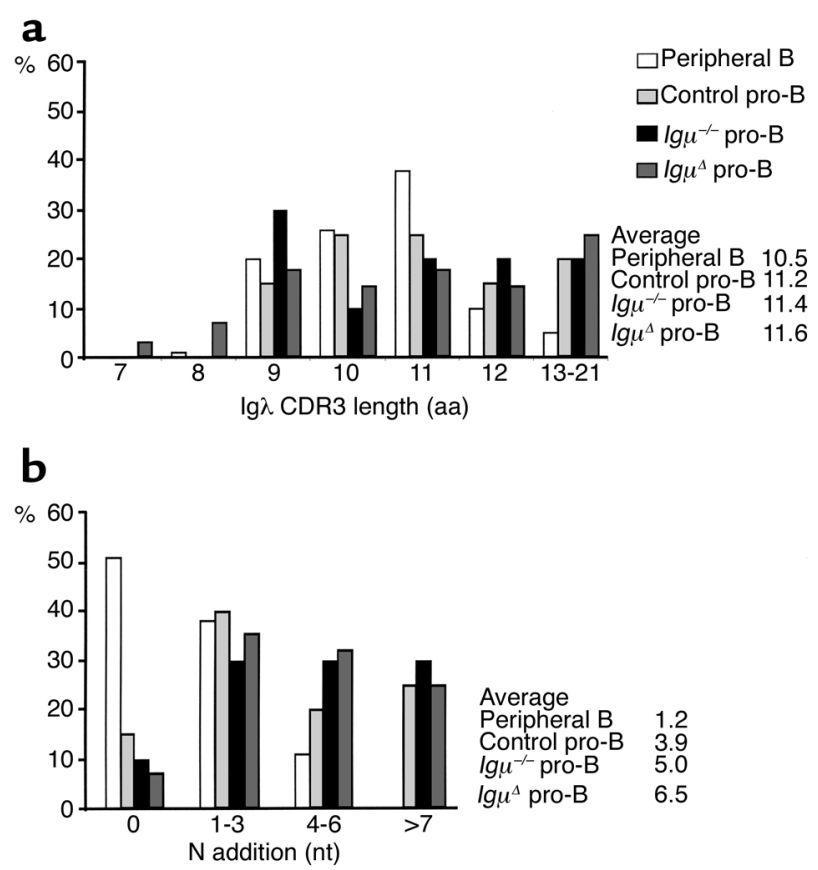
codons were enriched in control $\mathrm{CD} 34^{+} \mathrm{CD} 19^{+} \mathrm{B}$ cell progenitors, suggesting that an efficient selection process driven by pre-BCRs operates in normal $\mathrm{CD} 34^{+} \mathrm{CD} 19^{+} \mathrm{B}$ cell precursors. These Ig $\mu$-positive $\mathrm{B}$ cell precursors displaying surface pro-B cell markers are likely to be in transition to the pre- $\mathrm{B}$ cell stage and equivalent to the mouse $\mathrm{C}^{\prime}$ early pre-B cell fraction of Hardy's classification (58). By analogy to $\operatorname{Ig} \mu$ or $\operatorname{Ig} \beta$ knockout mice, the $C^{\prime}$ early pre$\mathrm{B}$ cell fraction is missing in $\mathrm{Ig} \mu^{--} \mathrm{CD} 34^{+} \mathrm{CD} 19^{+}$precursor B cells and results in a decrease of in-frame Ig $\mu$ rearrangements when compared with normal $\mathrm{CD} 34^{+} \mathrm{CD} 19^{+}$precursor $\mathrm{B}$ cells $(10,59)$.

In the mouse, $\psi \mathrm{L}$ has been implicated in IgH repertoire selection by virtue of pairing with some but not all $\mathrm{V}_{\mathrm{H}}$ domains $(16,18)$. Analysis of the $\mathrm{V}_{\mathrm{H}}$ repertoire in $\lambda 5^{-/-}$mice showed that the normal repertoire shift seen between the pro- $\mathrm{B}$ and the pre- $\mathrm{B}$ cell stage was absent (16). However, differences in pairing efficiency between $\psi \mathrm{L}$ and $\operatorname{IgH}$ are not likely to influence the selection against long or hydrophobic CDR3s in humans because these features are prevalent in control $\mathrm{CD} 34^{+} \mathrm{CD} 19^{+}$ cells, in immature $B$ cells that have passed $\psi \mathrm{L}$ selection, and in B cells that express $\psi$ Ls in the periphery $(31,49)$. A more likely explanation for selection against long and hydrophobic IgH CDR3s is that these features are associated with self-reactivity and might also interfere directly with IgH and IgL pairing $(31,49,60,61)$. Immature $\mathrm{B}$ cells displaying such Ab's therefore would be silenced by deletion or receptor editing, or alternatively, would fail to be positively selected in the mature B cell compartment (39, 40, 42-44, 62-64).

In the mouse, two Ig $\mu$-mediated mechanisms account for selection against self-reactive or poorly pairing Ab's during B cell development, receptor editing and deletion (39-44). Editing makes a major contribution to the $\mathrm{Ab}$ repertoire in mice: up to $25 \%$ of all $\mathrm{Ab}$ 's are produced by editing, but the role of deletion in repertoire selection is not known (65). Our experiments suggest that Ig $\mu$-mediated selection also makes a large contribution to shaping the human $\mathrm{Ab}$ repertoire. The selection against IgHs with long or hydrophobic CDR3s found in pro-B cells would require loss of at least $20-25 \%$ of all heavy chains.

In both mouse and human $B$ cells, $V(D) J$ recombination is generally ordered, starting with IgH rearrangement in pro-B cells followed by IgL rearrangement in pre-B cells $(66,67)$. However, analysis of mouse $\operatorname{Ig} \mu$ mutants and normal pro-B cells showed that IgL genes can recombine before IgH in pro-B cells $(54,68-71)$. Our experiments show that human control and $I g \mu$ deficient pro-B cells are similar to their mouse counterparts in that they undergo $\operatorname{Ig} \kappa$ and $\operatorname{Ig} \lambda$ gene rearrangements. These results are in agreement with the finding of rare $\operatorname{IgL}$ chain gene recombination in normal human pro-B cells and in Epstein-Barr virus-transformed fetal B cell precursors $(67,72)$. Thus, IgL rearrangement in the human is similar to the mouse in that it is not strictly dependent on Ig $\mu$ expression or pre-B cell development.
Up to $50 \%$ of human light chains in normal peripheral $\mathrm{B}$ cells show $\mathrm{N}$ nucleotide addition, but $\operatorname{IgK}$ or $\operatorname{Ig} \lambda$ CDR3s are never as long as 11 or 13 amino acids, respectively $(49,73-76)$. Ig $\kappa$ or $I g \lambda$ genes found in control and $I g \mu$-deficient pro-B cells differ from those found in normal peripheral B cells in that they show extensive $\mathrm{N}$ nucleotide addition associated with long IgL CDR3s that can reach up to 21 amino acids. Thus, long IgL CDR3s are produced in early developing B cells but they appear to be incompatible with B cell development and are deleted from the mature peripheral B cell repertoire.

$I g \mu$-deficient pro-B cells express $I g \kappa$ genes that display a bias to $3^{\prime} J \kappa s$ and $5^{\prime} V \kappa s$ consistent with persistent $V(D) J$ recombination. However, the bias to $3^{\prime} J \kappa s$ was incomplete since there was no significant increase in $J \kappa 5$, the most downstream $J \kappa$ segment. In addition, there was no bias to downstream $J \lambda$ s despite a genomic configuration that allows secondary rearrangements $(55,77,78)$. We speculate that in the absence of Ig $\mu$, human pro-B cells undergo several rounds of recombination on $I g \kappa$ but do not survive long enough to allow extensive secondary recombination. The absence of secondary recombination on $\operatorname{Ig} \lambda$ in $I g \mu$ deficient pro-B cells may result from a delayed recombination of this locus when compared with $\operatorname{Ig} \kappa(79$, 80). Alternatively, secondary recombination may be less efficient for $\operatorname{Ig} \lambda$ than $I g \kappa$.

Secondary $\operatorname{Ig} \kappa$ recombination is prominent in $I g \mu$ deficient pro-B cells yet not in normal control pro-B cells. Secondary recombination in Ig $\mu$-deficient pro-B cells therefore appears to be a default mechanism in the absence of Ig $\mu$ expression, and termination of secondary recombination in developing $B$ cells requires $\mathrm{BCR}$ signaling. We speculate that the regulation of Ig light chain gene recombination during $B$ cell development resembles that of T cell receptor- $\alpha$ (TCR- $\alpha$ ) chains during $\mathrm{T}$ cell development in that recombination is terminated by a yet to be determined positive selection signal transduced by the BCR (81). Developing $B$ cells remain in the pre-B cell compartment for a few hours whereas developing $\mathrm{T}$ cells remain in the $\mathrm{CD}^{+}{ }^{+} \mathrm{CD} 8^{+}$double-positive compartment for 3-4 days $(65,82,83)$. This kinetic difference may explain why Ig light chains are allelic excluded in B cells whereas TCR- $\alpha$ chains in T cells are not.

\section{Acknowledgments}

We thank M.J. Shlomchik and T.-A. Yang for comments on the manuscript and A. Deville, B. Lemmers, and J. Castro e Melo for help with bone marrow samples.

1. Pillai, S., and Baltimore, D. 1987. Formation of disulfide-linked $\mu 2 \omega 2$ tetramers in pre-B cells by the $18 \mathrm{k} \omega$-immunoglobulin light chain. Nature. 329:172-174.

2. Kerr, W.G., Cooper, M.D, Feng, L, Burrows, P.D, and Hendershot, L.M. 1989. Mu heavy chains can associate with a pseudo-light chain complex $(\Psi \mathrm{L})$ in human pre-B cell lines. Int. Immunol. 1:355-361.

3. Karasuyama, H., Kudo, A., and Melchers, F. 1990. The proteins encoded by the VpreB and lambda 5 pre-B cell-specific genes can associate with each other and with mu heavy chain. J. Exp. Med. 172:969-972.

4. Tsubata, T., and Reth, M. 1990. The products of pre-B cell-specific genes 
(lambda 5 and VpreB) and the immunoglobulin mu chain form a complex that is transported onto the cell surface. J. Exp. Med. 172:973-976

5. Hombach, J., Leclercq, L., Radbruch, A., Rajewsky, K., and Reth, M. 1988. A novel 34-kd protein co-isolated with the IgM molecule in surface IgM-expressing cells. EMBO J. 7:3451-3456.

6. Hermanson, G.G., Eisenberg, D., Kincade, P.W. and Wall, R. 1988. B29: a member of the immunoglobulin gene superfamily exclusively expressed on B-lineage cells. Proc. Natl. Acad. Sci. USA. 85:6890-6894.

7. Grawunder, U., et al. 1995. Down-regulation of RAG1 and RAG2 gene expression in preB cells after functional immunoglobulin heavy chain rearrangement. Immunity. 3:601-608.

8. Kitamura, D., Roes, J., Kuhn, R., and Rajewsky, K. 1991. A B cell deficient mouse by targeted disruption of the membrane exons of the immunoglobulin $\mu$ chain gene. Nature. 350:423-426.

9. Kitamura, D., et al. 1992. A critical role of $\lambda 5$ protein in B cell development. Cell. 69:823-831.

10. Gong, S., and Nussenzweig, M.C.. 1996. Regulation of an early developmental checkpoint in the B cell pathway by Ig beta. Science. 272:411-414.

11. Mundt, C., Licence, S., Shimizu, T., Melchers, F., and Martensson, I.L. 2001. Loss of Precursor B Cell Expansion but Not Allelic Exclusion in VpreB1/VpreB2 Double-deficient Mice. J. Exp. Med. 193:435-446.

12. Yel, L., et al. 1996. Mutations in the mu heavy-chain gene in patients with agammaglobulinemia. N. Engl.J. Med. 335:1486-1493.

13. Schiff, C., Lemmers, B., Deville, A., Fougereau, M., and Meffre, E. 2000 Autosomal primary immunodeficiencies affecting human bone marrow B cell differentiation. Immunol. Rev. 178:91-98.

14. Minegishi, Y., et al. 1998. Mutations in the human lambda5/14.1 gene result in B cell deficiency and agammaglobulinemia. J. Exp. Med. 187:71-77.

15. Minegishi, Y., et al. 1999. Mutations in Igalpha (CD79a) result in a complete block in B-cell development. J. Clin. Invest. 104:1115-1121.

16. ten Boekel, E., Melchers, F., and Rolink, A.G. 1997. Changes in the V(H) gene repertoire of developing precursor $\mathrm{B}$ lymphocytes in mouse bone marrow mediated by the pre-B cell receptor. Immunity. 7:357-368.

17. ten Boekel, E., Melchers, F., and Rolink, A.G. 1998. Precursor B cells showing $\mathrm{H}$ chain allelic inclusion display allelic exclusion at the level of pre-B cell receptor surface expression. Immunity. 8:199-207.

18. Wasserman, R., et al. 1998. A novel mechanism for B cell repertoire maturation based on response by $\mathrm{B}$ cell precursors to pre-B receptor assembly. J. Exp. Med. 187:259-264.

19. Kraj, P., et al. 1997. The human heavy chain Ig V region gene repertoire is biased at all stages of B cell ontogeny, including early pre-B cells. $J$. Immunol. 158:5824-5832.

20. Rao, S.P., et al. 1999. Biased VH gene usage in early lineage human $B$ cells: evidence for preferential Ig gene rearrangement in the absence of selection. J. Immunol. 163:2732-2740.

21. Milili, M., Schiff, C., Fougereau, M., and Tonnelle, C. 1996. The VDJ repertoire expressed in human preB cells reflects the selection of bona fide heavy chains. Eur. J. Immunol. 26:63-69.

22. Schroeder, H.W., Jr., Hillson, J.L., and Perlmutter, R.M. 1987. Early restriction of the human antibody repertoire. Science. 238:791-793.

23. Schroeder, H.W., Jr., and Wang, J.Y. 1990. Preferential utilization of conserved immunoglobulin heavy chain variable gene segments during human fetal life. Proc. Natl. Acad. Sci. USA. 87:6146-6150.

24. Hillson, J.L., et al. 1992. Emerging human B cell repertoire. Influence of developmental stage and interindividual variation. J. Immunol. 149:3741-3752.

25. Cuisinier, A.M., Gauthier, L., Boubli, L., Fougereau, M., and Tonnelle, C. 1993. Mechanisms that generate human immunoglobulin diversity operate from the 8 th week of gestation in fetal liver. Eur. J. Immunol. 23:110-118

26. Pascual, V., and Capra, J.D. 1991. Human immunoglobulin heavy-chain variable region genes: organization, polymorphism, and expression. Adv. Immunol. 49:1-74.

27. Huang, C., Stewart, A.K., Schwartz, R.S., and Stollar, B.D. 1992. Immunoglobulin heavy chain gene expression in peripheral blood B lymphocytes. J. Clin. Invest. 89:1331-1343.

28. Milner, E.C., Hufnagle, W.O., Glas, A.M., Suzuki, I., and Alexander, C. 1995. Polymorphism and utilization of human VH Genes. Ann. NY Acad. Sci. 764:50-61.

29. Brezinschek, H.P., Brezinschek, R.I., and Lipsky, P.E. 1995. Analysis of the heavy chain repertoire of human peripheral B cells using single-cell polymerase chain reaction. J. Immunol. 155:19-202.

30. Brezinschek, H.P., et al. 1997. Analysis of the human VH gene repertoire. Differential effects of selection and somatic hypermutation on human peripheral $\mathrm{CD} 5(+) / \operatorname{IgM}+$ and $\mathrm{CD} 5(-) / \operatorname{IgM}+\mathrm{B}$ cells. J. Clin. Invest. 99:2488-2501.

31. Raaphorst, F.M., Raman, C.S., Tami, J., Fischbach, M., and Sanz, I. 1997. Human Ig heavy chain CDR3 regions in adult bone marrow pre-B cells display an adult phenotype of diversity: evidence for structural selection of DH amino acid sequences. Int. Immunol. 9:1503-1515.
32. Dorner, T., et al. 1997. Analysis of the frequency and pattern of somatic mutations within nonproductively rearranged human variable heavy chain genes. J. Immunol. 158:2779-2789.

33. Shiokawa, S., et al. 1999. IgM heavy chain complementarity-determining region 3 diversity is constrained by genetic and somatic mechanisms until two months after birth. J. Immunol. 162:6060-6070.

34. Alt, F.W., et al. 1984. Ordered rearrangement of immunoglobulin heavy chain variable region segments. EMBO J. 3:1209-1219.

35. Reth, M., Petrac, E., Wiese, P., Lobel, L., and Alt, F.W. 1987. Activation of $\mathrm{V}$ kappa gene rearrangement in pre-B cells follows the expression of membrane-bound immunoglobulin heavy chains. EMBO J. 6:3299-3305.

36. Schlissel, M.S., and Baltimore, D. 1989. Activation of immunoglobulin kappa gene rearrangement correlates with induction of germline kappa gene transcription. Cell. 58:1001-1007.

37. Iglesias, A., Kopf, M., Williams, G.S., Buhler, B., and Kohler, G. 1991. Molecular requirements for the mu-induced light chain gene rearrangement in pre-B cells. EMBO J. 10:2147-2155.

38. Tsubata, T., Tsubata, R., and Reth, M. 1992. Crosslinking of the cell surface immunoglobulin (mu-surrogate light chains complex) on pre-B cells induces activation of $\mathrm{V}$ gene rearrangements at the immunoglobulin kappa locus. Int. Immunol. 4:637-641.

39. Nemazee, D.A., and Bürki, K. 1989. Clonal deletion of B lymphocytes in a transgenic mouse bearing anti-MHC class I antibody genes. Nature. 337:562-566.

40. Hartley, S.B., et al. 1991. Elimination from peripheral lymphoid tissues of self-reactive B lymphocytes recognizing membrane-bound antigens. Nature. 353:765-769.

41. Okamoto, M., et al. 1992. A transgenic model of autoimmune hemolytic anemia. J. Exp. Med. 175:71-79.

42. Tiegs, S.L., Russell, D.M., and Nemazee, D. 1993. Receptor editing in self-reactive bone marrow B cells. J. Exp. Med. 177:1009-1020.

43. Gay, D., Saunders, T., Camper, S., and Weigert, M. 1993. Receptor editing: an approach by autoreactive B cells to escape tolerance. J. Exp. Med. 177:999-1008.

44. Radic, M.Z., Erickson, J., Litwin, S., and Weigert, M. 1993. B lymphocytes may escape tolerance by revising their antigen receptors. J. Exp. Med. 177:1165-1173.

45. Yamagami, T., et al. 1999. Four of five RAG-expressing JCkappa-/small pre-BII cells have no L chain gene rearrangements: detection by high-efficiency single cell PCR. Immunity. 11:309-316.

46. Meffre, E., et al. 1996. A human non-XLA immunodeficiency disease characterized by blockage of B cell development at an early proB cell stage. J. Clin. Invest. 98:1519-1526.

47. Meffre, E., et al. 1997. A non-XLA primary deficiency causes the earliest known defect of B cell differentiation in humans: a comparison with an XLA case. Immunol. Lett. 57:93-99.

48. Fais, F., et al. 1998. Chronic lymphocytic leukemia B cells express restricted sets of mutated and unmutated antigen receptors. J. Clin. Invest. 102:1515-1525.

49. Meffre, E., et al. 2000. Circulating human B cells that express surrogate light chains and edited receptors. Nat. Immunol. 1:207-213.

50. Corbett, S.J., Tomlinson, I.M., Sonnhammer, E.L.L., Buck, D., and Winter, G. 1997. Sequence of the human immunoglobulin diversity (D) segment locus: a systematic analysis provides no evidence for the use of DIR segments, inverted D segments, "minor" D segments or D-D recombination. J. Mol. Biol. 270:587-597.

51. Kabat, E.A., Wu, T.T., Perry, H.M., Gottesman, K.S., and Foeller, C. 1991 Sequences of proteins of immunological interest. US Department of Health and Human Services. Bethesda, Maryland, USA. 2387 pp.

52. Desiderio, S.V., et al. 1984. Insertion of $\mathrm{N}$ regions into heavy-chain genes is correlated with expression of terminal deoxytransferase in B cells. Nature. 311:752-755.

53. Lafaille, J.J., DeCloux, A., Bonneville, M., Takagaki, Y., and Tonegawa, S. 1989. Junctional sequences of $\mathrm{T}$ cell receptor gamma delta genes: implications for gamma delta $\mathrm{T}$ cell lineages and for a novel intermediate of V-(D)-J joining. Cell. 59:859-870.

54. Bentolila, L.A., et al. 1999. Extensive junctional diversity in Ig light chain genes from early B cell progenitors of mu MT mice. J. Immunol. 162:2123-2128

55. Vasicek, T.J., and Leder, P. 1990. Structure and expression of the human immunoglobulin lambda genes. J. Exp. Med. 172:609-620.

56. Frippiat, J.P., et al. 1995. Organization of the human immunoglobulin lambda light-chain locus on chromosome 22q11.2. Hum. Mol. Genet. 4:983-991.

57. Williams, S.C., et al. 1996. Sequence and evolution of the human germline V lambda repertoire. J. Mol. Biol. 264:220-232.

58. Hardy, R.R., Carmack, C.E., Shinton, S.A., Kemp, J.D., and Hayakawa, K. 1991. Resolution and characterization of pro-B and pre-pro-B cell stages in normal mouse bone marrow. J. Exp. Med. 172:1213-1225.

59. Reichlin, A., et al. 2001. B cell development is arrested at the immature b cell stage in mice carrying a mutation in the cytoplasmic domain of immunoglobulin beta. J. Exp. Med. 193:13-24. 
60. Klonowski, K.D., Primiano, L.L., and Monestier, M. 1999. Atypical VH$\mathrm{D}-\mathrm{JH}$ rearrangements in newborn autoimmune MRL mice. J. Immunol. 162:1566-1572.

61. Schroeder, H.W., and Kirkham, P.M. 2000. Marriage, divorce, and promiscuity in human B cells. Nature Immunol. 1:187-188.

62. Sandel, P.C., and Monroe, J.G. 1999. Negative selection of immature B cells by receptor editing or deletion is determined by site of antigen encounter. Immunity. 10:289-299.

63. Gu, H., Tarlinton, D., Muller, W., Rajewsky, K., and Forster, I. 1991. Most peripheral B cells in mice are ligand selected. J. Exp. Med. 173:1357-1371.

64. Levine, M.H., et al. 2000. A B-cell receptor-specific selection step governs immature to mature B cell differentiation. Proc. Natl. Acad. Sci. USA 97:2743-2748.

65. Casellas, R., et al. 2001. Contribution of receptor editing to the antibody repertoire. Science. 291:1541-1544.

66. Blackwell, T.K., et al. 1989. Isolation of scid pre-B cells that rearrange kappa light chain genes: formation of normal signal and abnormal coding joins. EMBO J. 8:735-742.

67. Ghia, P., et al. 1996. Ordering of human bone marrow B lymphocyte precursors by single-cell polymerase chain reaction analyses of the rearrangement status of the immunoglobulin $\mathrm{H}$ and $\mathrm{L}$ chain loci.J. Exp. Med. 184:2217-2229.

68. Ehlich, A., et al. 1993. Immunoglobulin heavy and light chain genes rearrange independently at early stages of B cell development. Cell. 72:695-704.

69. Chen, J., et al. 1993. Immunoglobulin gene rearrangement in B cell deficient mice generated by targeted deletion of the JH locus. Int. Immunol. 5:647-656.

70. Grawunder, U., Haasner, D., Melchers, F., and Rolink, A. 1993 Rearrangement and expression of kappa light chain genes can occur without mu heavy chain expression during differentiation of pre-B cells. Int. Immunol. 5:1609-1618.

71. Novobrantseva, T.I., et al. 1999. Rearrangement and expression of immunoglobulin light chain genes can precede heavy chain expression during normal B cell development in mice. J. Exp. Med. 189:75-88.

72. Kubagawa, H., Cooper, M.D., Carroll, A.J., and Burrows, P.D. 1989. Light-chain gene expression before heavy-chain gene rearrangement in
pre-B cells transformed by Epstein-Barr virus. Proc. Natl. Acad. Sci. USA. 86:2356-2360

73. Klein, R., Jaenichen, R., and Zachau, H.G. 1993. Expressed human immunoglobulin kappa genes and their hypermutation. Eur. J. Immunol. 23:3248-3262.

74. Klein, U., Kuppers, R., and Rajewsky, K. 1993. Human IgM+IgD+ B cells, the major $\mathrm{B}$ cell subset in the peripheral blood, express $\mathrm{V}$ kappa genes with no or little somatic mutation throughout life. Eur. J. Immunol. 23:3272-3277.

75. Fischer, M., Klein, U., and Kuppers, R. 1997. Molecular single-cell analysis reveals that CD5-positive peripheral blood B cells in healthy humans are characterized by rearranged Vkappa genes lacking somatic mutation. J. Clin. Invest. 100:1667-1676.

76. Foster, S.J., Brezinschek, H.P., Brezinschek, R.I., and Lipsky, P.E. 1997. Molecular mechanisms and selective influences that shape the kappa gene repertoire of IgM+ B cells. J. Clin. Invest. 99:1614-1627.

77. Berinstein, N., Levy, S., and Levy, R. 1989. Activation of an excluded immunoglobulin allele in a human B lymphoma cell line. Science. 244:337-339.

78. Stiernholm, N.B., and Berinstein, N.L. 1993. Up-regulated recombination-activating gene expression in sIg-variants of a human mature $\mathrm{B}$ cell line undergoing secondary Ig lambda rearrangements in cell culture. Eur. J. Immunol. 23:1501-1507.

79. Hieter, P.A., Korsmeyer, S.J., Waldmann, T.A., and Leder, P. 1981. Human immunoglobulin kappa light-chain genes are deleted or rearranged in lambda-producing B cells. Nature. 290:368-372.

80. Korsmeyer, S.J., et al. 1981. Developmental hierarchy of immunoglobulin gene rearrangements in human leukemic pre-B-cells. Proc. Natl. Acad. Sci. USA. 78:7096-7100.

81. Borgulya, P., Kishi, H., Uematsu, Y., and Boehmer, H.V. 1992. Exclusion and inclusion of a and b T cell receptor alleles. Cell. 69:529-537.

82. Huesmann, M., Scott, B., Kisielow, P., and von Boehmer, H. 1991. Kinetics and efficacy of positive selection in the thymus of normal and $T$ cell receptor transgenic mice. Cell. 66:533-540.

83. Malissen, M., et al. 1988. A T cell clone expresses two T cell receptor alpha genes but uses one alpha beta heterodimer for allorecognition and self MHC-restricted antigen recognition. Cell. 55:49-59.

84. Lefranc, M.P. 2000. Locus maps and genomic repertoire of the human Ig genes. The Immunologist. 8:80-87. 